\title{
Bonobos, chimpanzees, gorillas, and orang utans use feature and spatial cues in two spatial memory tasks
}

\author{
Patricia Kanngiesser · Josep Call
}

Received: 17 April 2009 / Revised: 21 October 2009 / Accepted: 23 October 2009 / Published online: 13 November 2009

(C) The Author(s) 2009. This article is published with open access at Springerlink.com

\begin{abstract}
Animals commonly use feature and spatial strategies when remembering places of interest such as food sources or hiding places. We conducted three experiments with great apes to investigate strategy preferences and factors that may shape them. In the first experiment, we trained 17 apes to remember 12 different food locations on the floor of their sleeping room. The 12 food locations were associated with one feature cue, so that feature and spatial cues were confounded. In a single test session, we brought the cues into conflict and found that apes, irrespective of species, showed a preference for a feature strategy. In the second experiment, we used a similar procedure and trained 25 apes to remember one food location on a platform in front of them. On average, apes preferred to use a feature strategy but some individuals relied on a spatial strategy. In the final experiment, we investigated whether training might influence strategy preferences. We tested 21 apes in the platform set-up and found that apes used both, feature and spatial strategies irrespective of training. We conclude that apes can use feature and spatial strategies to remember the location of hidden food items, but that task demands (e.g. different numbers of search locations) can influence strategy preferences. We found no evidence, however, for the role of training in shaping these preferences.
\end{abstract}

P. Kanngiesser $(\bowtie)$

Department of Experimental Psychology,

University of Bristol, 12a Priory Road,

Bristol BS8 1TU, United Kingdom

e-mail: patricia.kanngiesser@bristol.ac.uk

J. Call

Department of Developmental and Comparative Psychology,

Max Planck Institute for Evolutionary Anthropology,

Deutscher Platz 6, 04103 Leipzig, Germany
Keywords Spatial memory $\cdot$ Cues $\cdot$ Spatial strategies · Great apes

\section{Introduction}

When animals visit food sources, avoid possible areas of danger or seek refuge they often rely on memories to identify such places of interest. In order to form memories of different places, animals can either exploit cues in the environment, such as odours, colours, and landmarks, or use internal cues such as proprioceptive cues (Shettleworth 1998). In general, cues in the environment can be exploited by using one of two strategies. Spatial strategies rely on cues that are based on the spatial layout of a place like its geometry or its relation to a configuration of landmarks, whereas feature strategies rely on cues that are based on specific features (colours, patterns, shapes) of objects that are contiguous with a place of interest.

There is ample evidence that animals use feature and spatial strategies to remember places of interest, often, however, they appear to use one of the two strategies preferentially. Thus, while some species like toads, pigeons, dogs, and lizards were shown to prefer spatial strategies (Williams 1967; Strasser and Bingman 1996; Dumas 1998; Day et al. 2003), other species like chicks and goldfish were shown to prefer feature strategies (Vallortigara et al. 1990; Vargas et al. 2004). On some occasions, members of closely related species or even of the same species showed differences in strategy preferences. These differences have been attributed to ecological factors (storing vs. non-storing bird species: Clayton and Krebs 1994; Brodbeck 1994; Brodbeck and Shettleworth 1995), sex differences (shiny cowbirds: Astié et al. 1998; chicks: Vallortigara 1996), or hemispheric differences (i.e. the brain hemisphere in charge 
of solving a task) (chicks: Vallortigara 2000; Regolin et al. 2005). Similarly, differences in the encoding of spatial information in humans have been attributed to sex differences and differences in hormone levels (i.e. in relation to the menstrual cycle in females) (Postma et al. 1999, 2004).

Haun et al. (2006) used a simple food search task to investigate search strategies in great apes and 1- and 3-yearold children. In the task, the participants were first shown a reward being hidden under one of three (or two) containers. Then, out of the participants' sight, the positions of the containers were switched. The reward either moved with the container under which it was hidden to a new location or stayed in the same location but under a container with different features. One-year-old children and great apes performed better when the reward stayed in the same location (spatial strategy), while 3-year-old children performed better when the reward moved with the container (feature strategy). The authors argued that the great apes and 1-year-old children share a bias for the spatial strategy, which is overcome in the course of child development (i.e. at about 3 years) - maybe due to the acquisition of language. Likewise, Tinklepaugh (1932) found reliance on spatial cues in two chimpanzees, whose performance in retrieving a reward from a set of different containers was more affected when the reward moved with the containers than when the reward stayed in the same location but under a different container. Although this evidence suggests that the great apes preferentially rely on a spatial strategy, one may wonder how flexible apes are in overcoming this bias. Would great apes for example switch to using a feature-based strategy under circumstances that favoured the use of such a strategy?

Several studies investigating strategy use during spatial reorientation have documented that animals and human children are able to overcome biases for one strategy and use multiple strategies (see Cheng and Newcombe 2005, for a review). In spatial reorientation tasks, animals or humans have to remember the location of a target in one corner of a rectangular room, which often contains a feature cue such as a coloured wall or a small marker. After the participants are disoriented, they have to reorient themselves in order to find the target location. They can find the location by using either the spatial cue (the geometry of the room) or the feature cue (the coloured wall/ marker) or by combining both cues. Twenty-month-old children and rats were shown to use the geometry of the room to reorient while ignoring the feature cue (Cheng 1986; Hermer and Spelke 1994), whereas human adults, rhesus monkeys, pigeons, fish, and chicks were all shown to be able to conjoin both cues (Hermer and Spelke 1994; Kelly et al. 1998; Gouteux et al. 2001; Sovrano et al. 2003; Vallortigara et al. 2005). However, if the size of the search space was increased, 2- to 3-year-old children were able to use feature cues in addition to geometric information during reorientation (Learmonth et al. 2002). Two opposing explanations have been offered to explain these findings. On one view, children and other animals use a geometric process to reorient and an associate process to link feature cues to specific locations (Lee et al. 2006). On another view, the relative salience of the feature cues was enhanced through increasing the size of the search space as more distal markers might be perceived as more stable than proximal markers (see e.g. Newcombe and Ratliff 2007, for a discussion). Irrespective of the exact process at play, these findings indicate that the modifications of the task environment might lead to a change in strategy preferences.

The question remains whether great apes would be able to switch to using a feature strategy if they were presented with a task where the salience of the feature cues was enhanced. We aimed at increasing the salience of the feature cues by two different measures: first, by increasing the number of search locations, and second, by making all target locations contiguous with one feature cue (i.e. one type of container that covered the food that was hidden in the target locations). By using a medium-scale space $\left(3 \mathrm{~m}^{2}\right)$, in which animals could move around and exploit baited locations, we also tried to create a more ecologically valid setting.

In our first experiment, we trained animals from all four great ape species to search for hidden food items under containers on the floor of their sleeping rooms. During the training phase the apes had to learn to find food items in the same locations under the same kind of containers, so that the feature and the spatial cues were confounded. In order to evaluate which strategy they used, we brought the two cues into conflict by switching the positions of rewarded containers with positions of unrewarded containers.

We conducted a second experiment to evaluate whether apes would switch back to using a spatial strategy if the number of search locations was reduced. We used a task that was similar to the one used by Haun et al. (2006). In our version of the task, the apes had to learn the location of a food reward that was hidden under one of three containers on a platform in front of them. After the apes did so successfully, we tested them again with conflicting cues by switching the positions of the containers on the platform.

In a final experiment, we investigated why the findings of our second experiment differed from those of Haun et al. (2006). We hypothesized that this may be due to fact that we had trained apes to remember the food location prior to testing them with conflicting cues. In order to investigate this hypothesis, we used a between-subjects design with three experimental groups. One experimental group was tested with the same design as in Experiment 2, a second group with a design very similar to the one used by Haun et al. (2006), and a third group with a combination of the two designs. 


\section{Experiment 1}

The goal of Experiment 1 was to investigate which search strategy great apes would use when the salience of the feature cues was increased. We first trained apes to search for food items under containers on the floor of their sleeping room and then brought feature and spatial cues into conflict in a single test session. Eleven subjects first participated in Experiment 1 and then in Experiment 2, and six subjects first participated in Experiment 2 and then in Experiment 1.

\section{Methods}

\section{Subjects}

We tested 17 great apes (4 bonobos, 3 gorillas, 5 orang utans, 5 chimpanzees - see Table 1 for details) that were housed at the Wolfgang Köhler Primate Research Center at the Zoo Leipzig. The apes had access to indoor and outdoor enclosures that were furnished with climbing trees, ropes, and different kinds of enrichment devices. They were fed four times a day with a diet of vegetables and fruits and had ad libitum access to water. The experiment was conducted between 9 am and $1 \mathrm{pm}$ after the apes had been fed their morning ration of fruits and vegetables.

\section{Materials}

For each subject, we used 36 containers. For some subjects, we used 12 metallic, heart-shaped containers and 24 round, flat containers with green and white stripes. For other subjects we used 12 metallic, heart-shaped containers and 24 round, flat containers with red and white stripes. We distributed the 36 containers evenly on the floor of the subject's sleeping room in an area of $3 \mathrm{~m}^{2}$ (see Fig. 1). All the sleeping room floors were covered with tiles (tile size $19.5 \mathrm{~cm} \times 19.5 \mathrm{~cm}$ ), and we used the tiles on the floor as markers, so that we could always distribute the containers in the same pattern.

\section{Procedure}

Initially, all subjects received three training sessions per day, during which we placed banana slices as rewards under the 12 heart-shaped containers (with the exception of one subject Bimbo, who received pellets as rewards). During training, the 36 containers ( 12 heart-shaped and 24 flat round containers) remained in the same pattern on the floor, so that the place and the feature cues did not change and were always confounded (see Fig. 1). In each session, the subjects entered the room from the same side (i.e. always on the right side in relation to the set-up, or always on the left side in relation to the set-up). Subjects could then turn over the containers and search for the hidden food items under the containers. A session ended once subjects had discovered all hidden food items. We terminated the training if subjects succeeded in finding food in 10 of their first 12 searches in at least 2 of 3 sessions on 1 day.

The test phase consisted of a single test day with two training sessions and one test session, which directly followed the second training session. If the subjects completed both training sessions successfully (10 of their first 12 searches correct), we conducted the test session. If subjects did not complete the two training sessions successfully, they received another training session, and the test phase was repeated on the following day. During the test session, the pattern of the containers on the floor was changed that is the positions of the heart-shaped containers and the flat round containers were switched. The 12 heart-shaped containers now occupied 12 of the 24 positions that were previously occupied by the flat round containers, and the flat round containers occupied all positions that were previously occupied by the heart-shaped containers. During the test session, we baited all containers so that neither the place nor the feature cues were differentially reinforced. The subjects were allowed to search all containers and a session usually ended once subjects either had discovered all hidden food items or discontinued their search for approximately $1 \mathrm{~min}$.

\section{Data scoring and analysis}

We used live coding and coding from videotapes to determine which containers were searched by the subjects. If subjects turned the containers or lifted them off the floor, they were coded as searched containers. During the subjects' first 12 searches in the test session, the following dependent variables were coded: searches under heartshaped containers (feature searches), searches under round, flat containers that were in previously baited locations (place searches), and searches under round, flat containers in locations that were never baited (control searches).

As the data did not fulfil some of the requirements for using parametric statistics, all analyses were conducted with non-parametric tests. First, we tested for species effects using Kruskal-Wallis $H$ tests. We then assessed which strategy the subjects used on average by comparing the percentages of feature and place searches to chance using one-sample Wilcoxon tests. Chance was defined as conducting the three possible searches (feature, place and control) with a probability of 0.33 each. Finally, we analysed the individual data and evaluated whether subjects searched the containers randomly or whether they preferred to use either a feature or a spatial strategy. Preference for the use of one of the strategies was assessed by comparing the number of feature and place searches to chance $(0.33)$ 
Table 1 List of apes that participated in Experiments 1-3

\begin{tabular}{|c|c|c|c|c|c|c|c|c|c|c|c|c|}
\hline \multirow[t]{2}{*}{ Species } & \multirow[t]{2}{*}{ Name } & \multirow[t]{2}{*}{ Sex } & \multirow[t]{2}{*}{ Age } & \multirow[t]{2}{*}{ Order } & \multicolumn{2}{|c|}{ Experiment 1} & \multicolumn{3}{|c|}{ Experiment 2} & \multicolumn{3}{|l|}{ Experiment 3} \\
\hline & & & & & $\begin{array}{l}\% \\
\text { Feature }\end{array}$ & $\begin{array}{l}\% \\
\text { Place }\end{array}$ & Group & $\begin{array}{l}\% \\
\text { Feature }\end{array}$ & $\begin{array}{l}\% \\
\text { Place }\end{array}$ & Group & $\begin{array}{l}\% \\
\text { Feature }\end{array}$ & $\begin{array}{l}\% \\
\text { Place }\end{array}$ \\
\hline Bonobo & Joey & $\mathrm{m}$ & 24 & E1, E2 & 58 & 25 & Exp & 0 & $92 *$ & Learning & $100 *$ & 0 \\
\hline Bonobo & Kuno & $\mathrm{m}$ & 10 & $\mathrm{E} 1, \mathrm{E} 2$ & $92 *$ & 0 & Exp & $100 *$ & 0 & Learning \& seeing & $100^{*}$ & 0 \\
\hline Bonobo & Limbuko & $\mathrm{m}$ & 11 & $\mathrm{E} 1, \mathrm{E} 2$ & $92 *$ & 0 & $\operatorname{Exp}$ & $92 *$ & 8 & Seeing & 25 & 50 \\
\hline Bonobo & Ulindi & $\mathrm{f}$ & 13 & $\mathrm{E} 2$ & - & - & Exp & $100 *$ & 0 & Learning & 58 & 42 \\
\hline Bonobo & Yasa & $\mathrm{f}$ & 9 & E2, E1 & $92 *$ & 0 & Exp & $100 *$ & 0 & Learning \& seeing & $92 *$ & 8 \\
\hline Chimpanzee & Annett & $\mathrm{f}$ & 8 & - & - & - & - & - & - & Seeing & $75^{*}$ & 25 \\
\hline Chimpanzee & Corry & $\mathrm{f}$ & 30 & E2 & - & - & Ctrl & 33 & $67 *$ & Learning \& seeing & 0 & $100 *$ \\
\hline Chimpanzee & Dorien & f & 26 & E2 & - & - & Exp & 33 & $67 *$ & Seeing & 0 & $100 *$ \\
\hline Chimpanzee & Fifi & $\mathrm{f}$ & 15 & - & - & - & - & - & - & Learning & 50 & 0 \\
\hline Chimpanzee & Fraukje & $\mathrm{f}$ & 31 & E2 & - & - & Ctrl & $100 *$ & 0 & Learning \& seeing & 17 & $83 *$ \\
\hline Chimpanzee & Frodo & $\mathrm{m}$ & 13 & E2 & - & - & Ctrl & $100^{*}$ & 0 & Learning & $100^{*}$ & 0 \\
\hline Chimpanzee & Gertruida & $\mathrm{f}$ & 14 & E2, E1 & $100 *$ & 0 & Exp & $92 *$ & 8 & Seeing & 8 & $67 *$ \\
\hline Chimpanzee & Jahaga & $\mathrm{f}$ & 14 & E1, E2 & $100 *$ & 0 & Exp & $100^{*}$ & 0 & Seeing & $100^{*}$ & 0 \\
\hline Chimpanzee & Lome & $\mathrm{m}$ & 6 & E1, E2 & $75^{*}$ & 0 & Exp & 42 & 17 & Learning & 8 & 50 \\
\hline Chimpanzee & Natascha & $\mathrm{f}$ & 28 & - & - & - & - & - & - & Seeing & 17 & 42 \\
\hline Chimpanzee & Patrick & $\mathrm{m}$ & 10 & $\mathrm{E} 2, \mathrm{E} 1$ & $83 *$ & 0 & Exp & $100 *$ & 0 & Learning \& seeing & $100 *$ & 0 \\
\hline Chimpanzee & Pia & f & 8 & - & - & - & - & - & - & Seeing & 8 & 42 \\
\hline Chimpanzee & Riet & $\mathrm{f}$ & 30 & - & - & - & - & - & - & Learning & 0 & $100 *$ \\
\hline Chimpanzee & Robert & $\mathrm{m}$ & 31 & E2 & - & - & Ctrl & $100 *$ & 0 & Seeing & 0 & $100^{*}$ \\
\hline Chimpanzee & Sandra & $\mathrm{f}$ & 14 & E1, E2 & $78^{*}$ & 0 & Exp & $92 *$ & 8 & Learning & 17 & $83^{*}$ \\
\hline Chimpanzee & Tai & f & 5 & - & - & - & - & - & - & Learning & 0 & $100 *$ \\
\hline Chimpanzee & Ulla & $\mathrm{f}$ & 30 & E2 & - & - & Ctrl & $100 *$ & 0 & - & - & - \\
\hline Gorilla & Bebe & f & 27 & $\mathrm{E} 2, \mathrm{E} 1$ & $83 *$ & 0 & Exp & $67 *$ & 8 & - & - & - \\
\hline Gorilla & Kibara & f & 3 & E1, E2 & $75^{*}$ & 8 & Exp & 0 & $100 *$ & - & - & - \\
\hline Gorilla & Viringika & f & 12 & E1, E2 & $100 *$ & 0 & Exp & 0 & 33 & - & - & - \\
\hline Orang Utan & Bimbo & $\mathrm{m}$ & 26 & E1, E2 & $100 *$ & 0 & Exp & 0 & $100 *$ & - & - & - \\
\hline Orang Utan & Dokana & $\mathrm{f}$ & 18 & E2, E1 & $91 *$ & 9 & Exp & $100 *$ & 0 & - & - & - \\
\hline Orang Utan & Dunja & $\mathrm{f}$ & 34 & E2 & - & - & Exp & 25 & 58 & - & - & - \\
\hline Orang Utan & Kila & $\mathrm{f}$ & 7 & E1, E2 & $73 *$ & 9 & Exp & $92 *$ & 8 & - & - & - \\
\hline Orang Utan & Padana & $\mathrm{f}$ & 9 & $\mathrm{E} 2, \mathrm{E} 1$ & $92 *$ & 0 & Exp & $100 *$ & 0 & - & - & - \\
\hline Orang Utan & Pini & $\mathrm{f}$ & 19 & E1, E2 & $100 *$ & 0 & Exp & 0 & $100 *$ & - & - & - \\
\hline
\end{tabular}

The column 'order' gives the order in which subjects participated in Experiment 1 (E1) and Experiment 2 (E2). 'exp' indicates that apes belonged to the experimental group and 'ctrl' indicates that they belonged to the control group. 'learning' indicates that apes were trained prior to the test, 'learning \& seeing' indicates that apes were trained and observed the reward being hidden, and 'seeing' indicates that apes were not trained and only observed the reward being hidden. Asterisks indicate significant $(P \leq 0.05)$ deviation from chance according to two-tailed binomial test

using binomial tests. All statistical tests were evaluated with a significance level of 0.05 and exact, two-tailed $P$ values are reported where applicable.

\section{Results}

On average, the subjects needed 7.2 \pm 3.5 (Mean $\pm \mathrm{SE}$ ) sessions to reach the training criterion. In the first training session, the subjects searched the heart-shaped containers on average $5.5 \pm 3.5$ (Mean $\pm \mathrm{SE}$ ) times in their first 12 searches. Only two subjects (Sandra, Trudi) searched under the heart-shaped container significantly above chance (according to binomial tests with chance level 0.33) during their first 12 searches. This indicates that the majority of the apes had no initial preference for the heart-shaped containers over the flat, round containers.

Figure 2 depicts the mean percentage of feature, place and control searches in the test session for all four ape species. We found no significant differences between species with respect to the percentages of feature and place searches (Kruskal-Wallis $H$ tests: feature: $\chi^{2}=0.426$, $\mathrm{df}=3, P=0.946$; place: $\left.\chi^{2}=2.209, \mathrm{df}=3, P=0.595\right)$. On 


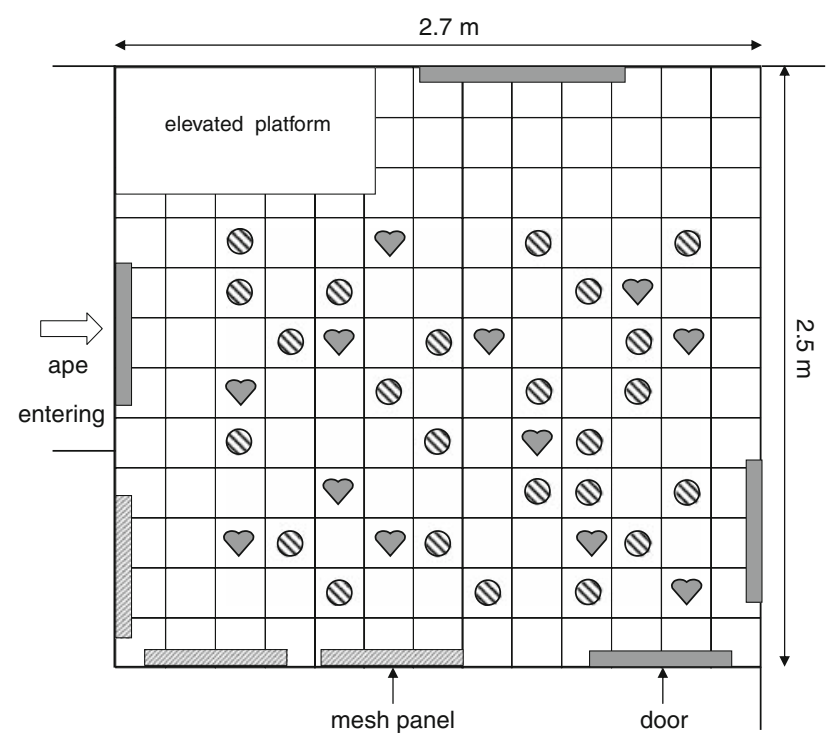

Fig. 1 Schematic of one of the sleeping rooms (chimpanzee sleeping room) and the set-up used in Experiment 1. Hearts indicate heartshaped containers and striped circles indicate round, flat containers

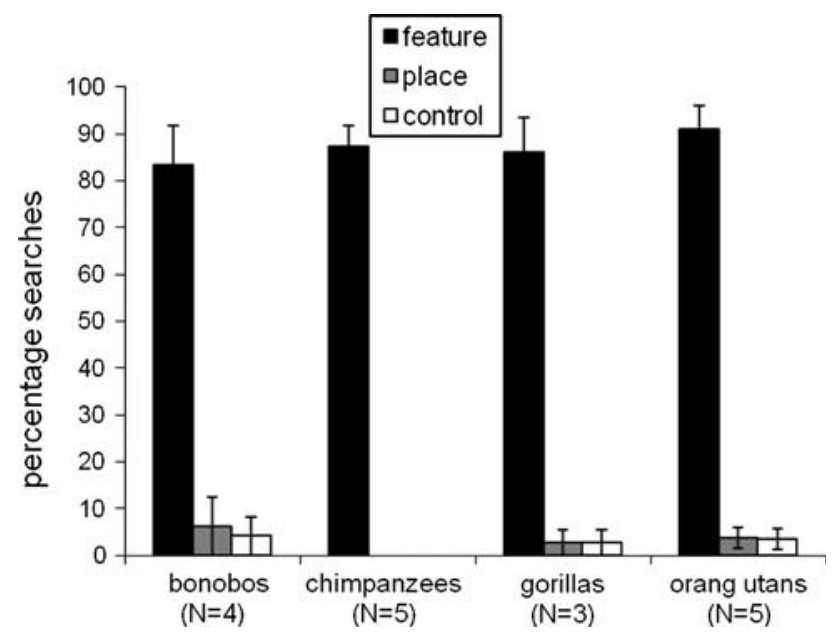

Fig. 2 Mean percentage of feature, place and control searches $( \pm$ SE) per species in Experiment 1

average, the subjects made feature searches significantly above chance (Wilcoxon test: $Z=3.638, P<0.001$ ) and place searches significantly below chance $(Z=3.822$, $P<0.001)$. Focusing on individual data, we found that all subjects except for one bonobo conducted feature searches significantly above chance (see Table 1 ). In addition, all subjects made feature searches in their first search attempt. Thus, apes, irrespective of species, relied on a feature strategy during their search.

\section{Discussion}

We found that subjects from all four great ape species preferentially used a feature strategy to search for hidden food items on the floor of their sleeping room. After having been trained to find food in different locations under identical containers, all subjects except for one bonobo used the feature strategy when they were tested with conflicting cues. In addition, all subjects demonstrated use of feature cues during their first search. As we found no difference in performance between the four great ape species, these results provide us with a strong indication that, with respect to our task, all four great apes species show a preference for using the feature strategy.

In contrast to earlier studies (Tinklepaugh 1932; Haun et al. 2006), that demonstrated that apes preferred a spatial strategy when remembering the location of a food reward, we found in our experiment that they exclusively used a feature strategy. Thus, apes seem to be able to switch between different strategies depending on the demands of the task. Our results suggest that the present task facilitated the use of a feature strategy-possibly through increasing the salience of the feature cues or through taxing memory by increasing the number of search locations.

In order to further investigate the influence of different tasks on strategy preferences, a second experiment was conducted in which subjects were tested with only three search locations on a platform in front of them. As this setup was similar to the one used by Haun et al. (2006), we predicted that the apes would show a preference for using a spatial strategy.

\section{Experiment 2}

The goal of Experiment 2 was to investigate which strategy great apes would use if the size of the test space and the number of search locations were reduced. We thus trained great apes from all four species to find food under one of three containers on a small platform in front of them (as described by Haun et al. 2006). In the test session, we then brought the feature and spatial cues in conflict by switching the locations of the containers. We used three containers only one of which was distinct from the other two containers, which was the same property of containers as in Experiment 1. We opted for this set-up rather than presenting three containers in a medium-scale space to make the experiment more comparable to Haun et al. (2006). In addition, we wanted to investigate whether the number of distinct containers would have an influence on which strategy subjects used. Thus, we introduced a control group that was presented with three distinct containers instead of two identical containers and one distinct one (like the experimental group). 


\section{Methods}

\section{Subjects}

The same subjects as in Experiment 1 were tested. Eight additional subjects (1 bonobo, 1 orang utan, 6 chimpanzees) participated in the current experiment, so that, in total, 25 subjects were tested (see Table 1 for details). Five of the 25 subjects ( 5 chimpanzees) belonged to the control group. Subjects from the other great ape species were not part of the control group, as the sample sizes were too small.

\section{Materials}

For the experimental group, we used containers that differed in shape, colour, and size: two blue cups, two green cups, one blue shell-shaped sand mould, and one red turtleshaped sand mould (see Fig. 3a). The subjects were presented with three containers in total, two of which were identical cups and one of which was a sand mould (i.e. two green cups and a blue shell-shaped sand mould, two green cups and a red turtle-shaped sand mould, or two blue cups and a red turtle-shaped sand mould). For the control group, we used three distinct containers: a green cup, a blue square-shaped container, and a red turtle-shaped sand mould (see Fig. 3a).

\section{Procedure}

We used the same procedure for the experimental and the control group. Initially, the subjects received one training session per day with 12 trials per session. During training, we aligned a set of three containers on a $40 \mathrm{~cm}$ wide and $70 \mathrm{~cm}$ long platform in front of the apes with approximately $20 \mathrm{~cm}$ between individual containers (see Fig. 4a). The containers remained in the same locations on the platform, and we always baited the same container with a banana slice as reward (with the exception of two subjects Bimbo and Fraukje, who received half a pellet). In the
Fig. 3 Pictures of the containers used to cover the reward. a shows the containers that were used for the experimental and the control group in Experiment 2 and $\mathbf{b}$ the containers that were used in Experiment 3
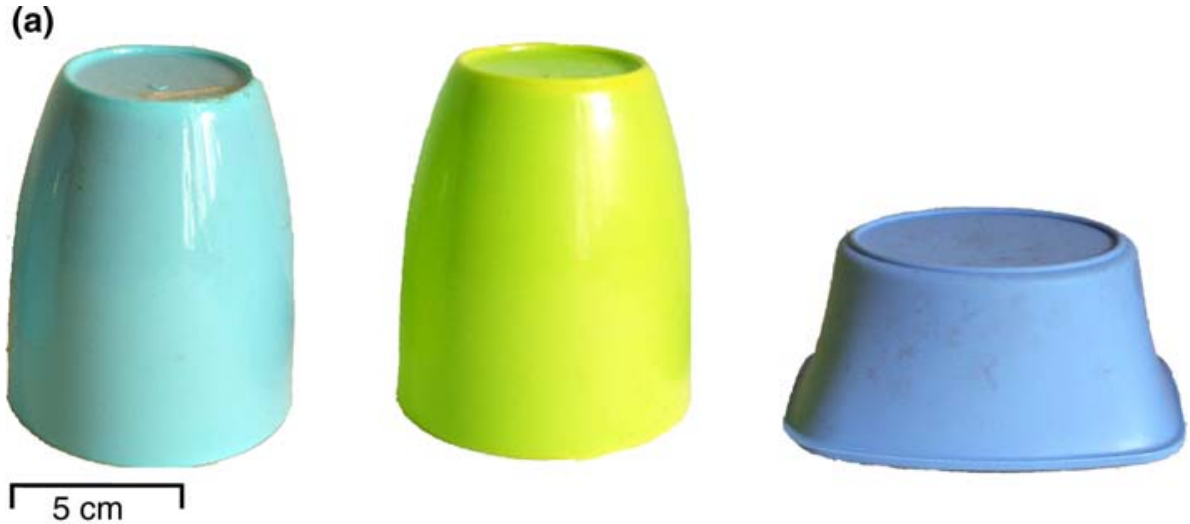

Experiment 2

(b)

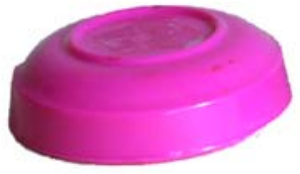

$5 \mathrm{~cm}$

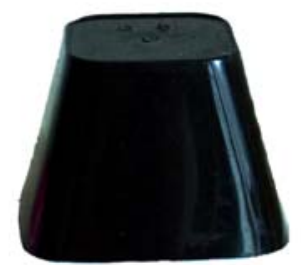

Experiment 3 


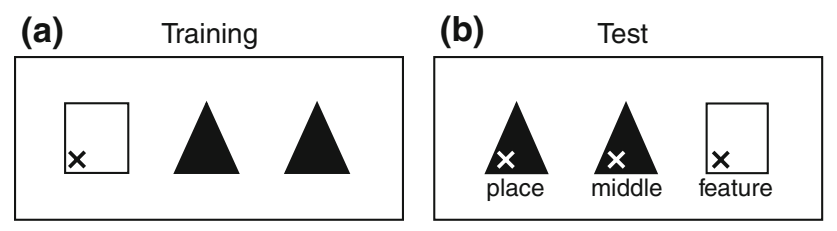

Fig. 4 Schematic of the set-up used for the experimental group in Experiment 2. Squares and triangles indicate containers that differ in shape, size, and colour, the cross indicates the reward position. $\mathbf{a}$ depicts a typical set-up in the training phase, and $\mathbf{b}$ depicts a typical set-up in the test phase. In the training phase, only the distinct container (i.e. the square in the schematic) was baited with a food reward; in the test phase, all containers were baited. 'Place', 'middle', and 'feature' indicate how choices of the respective containers were coded

experimental group, we always baited the distinct container (the sand mould) and in the control group we baited one of the three distinct containers (but a different one for each ape). Recall that all containers were different in the control group. In both groups, we presented half of the subjects from one species with the baited container on the subjects' right-hand side of the platform and the other half with the baited container on the subjects' left-hand side of the platform.

At the beginning of each training trial, we lowered an opaque plastic panel (occluder) in front of the containers to conceal the baiting process. We first showed the subjects the reward and then baited the respective container behind the occluder. We made sure to touch and move each container to ensure that the subjects could not use any auditory or spatial cues from the baiting process to find the food. The baiting process lasted about $10 \mathrm{~s}$, after which we raised the occluder, and the subjects were allowed to choose containers by pointing at them through holes in a Plexiglas panel. We lifted each container the subjects pointed at and laid it open on the platform. The subjects could thus make up to three choices and request all containers to be opened. In case a food item was hidden under the container, we gave it to the subjects through the hole in the middle of the panel. The training phase was completed once the subjects correctly chose (i.e. first chose) the baited container in at least 10 of 12 choices during one session.

The test phase consisted of a single test day with 6 training trials and 12 test trials, which directly followed the last training trial. First, the subjects received 6 training trials and if they made at least 5 correct choices, they received 12 test trials. If they made less than five correct choices, they received additional six training trials, and the test phase was repeated on the following day.

The procedure in the test session was identical to the one in training sessions with the following exceptions. (1) In the first trial, we moved the baited container behind the occluder to the opposite side, where it stayed for the remaining trials (see Fig. 4b). (2) We baited all containers out of sight of the subjects. (3) Subjects were allowed to make only one choice. These changes ensured that: (1) the feature and the place cue conflicted, (2) that neither the feature nor the place cue were differentially reinforced, and (3) that the subjects could not discover that all containers were baited.

\section{Data scoring and analysis}

We used live coding and coding from videotapes to score the following dependent variables: choices of the container under which the food could previously be found (feature choice), choices of the container in the place where the food could be found during training (place choice), or choices of the container in the middle of the platform (middle choice) (see Fig. 4b).

We analysed the data of the experimental group with the same non-parametric tests as in Experiment 1. In addition, we investigated whether sex had an influence on feature and place choices using Mann-Whitney $U$ tests and whether age correlated with feature and place choices, respectively, using Spearman rank correlations. We also analysed the first choices of the subjects in the experimental group for species differences and preferences for one of the three categories of choice (feature, place, middle) using chi-square tests. Furthermore, we compared the performance of the chimpanzees of the experimental and of the control group by means of Mann-Whitney $U$ tests, in order to assess whether the number of distinct containers had an influence on which strategies apes used preferentially. In addition, we investigated whether there was a significant change in strategy preference from Experiment 1 to Experiment 2 by comparing the percentages of feature and place choices of Experiment 1 and Experiment 2 using Wilcoxon tests. We further explored whether feature and place choices on the platform were affected by the order in which subjects completed Experiments 1 and 2 using Mann-Whitney $U$ tests (Note that we also included subjects in the analysis, which participated in Experiment 2, but never participated in Experiment 1). We conducted the same analysis to explore whether performance in Experiment 1 was affected by the order in which subjects participated in the two experiments. All statistical tests were evaluated with a significance level of 0.05 and exact, two-tailed $P$ values are reported where applicable.

\section{Results}

The subjects needed on average $2.1 \pm 1.3$ sessions (Mean $\pm \mathrm{SE}$ ) to reach the training criterion. Figure 5 depicts the mean percentage of choices in the test session for all four great ape species. We found no significant differences between species with respect to the percentages 


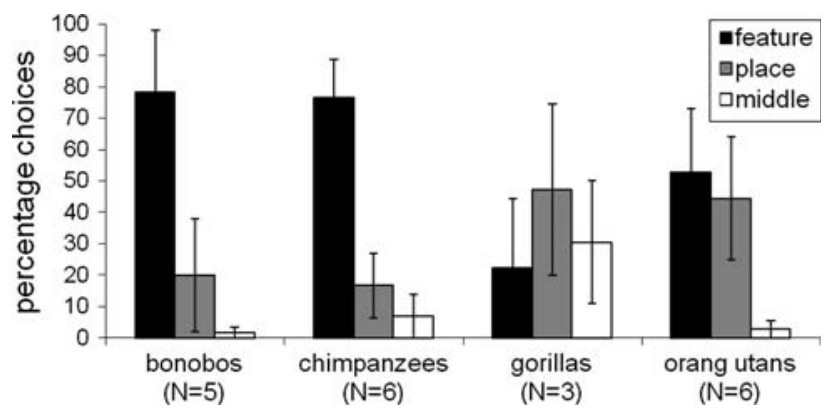

Fig. 5 Mean percentage of feature, place and middle choices $( \pm \mathrm{SE})$ per species in Experiment 2

of feature and place choices (Kruskal-Wallis $H$ tests: feature: $\chi^{2}=4.371, \quad \mathrm{df}=3, \quad P=0.234 ;$ place: $\chi^{2}=2.955$, $\mathrm{df}=3, P=0.420$ ). We also found no differences in feature and place choices between males and females (MannWhitney $U$ tests: feature: $N=20, U=38.500, P=0.787$; place: $N=20, U=38.000, P=0.756$ ), nor an influence of age on feature and place choices (Spearman rank correlations: feature: $N=20$, rho $=-0.330, P=0.156$; place: $N=20$, rho $=0.323, P=0.165)$. On average, the subjects made feature choices significantly above chance (Wilcoxon tests: feature: $Z=2.684, P=0.006$; place: $Z=0.367$, $P=0.723)$.

Focusing on individual data, we found that 12 subjects (4 bonobos, 4 chimpanzees, 1 gorilla, 3 orang utans) made feature choices, 5 subjects ( 1 bonobo, 1 chimpanzee, 1 gorilla, 2 orang utans) made place choices and 1 subject (1 gorilla) made middle choices significantly above chance (see Table 1). Two subjects ( 1 chimpanzee, 1 orang utan) made none of the choices significantly above chance. Thus, on average subjects preferred to use a feature strategy, yet some individuals preferred to use a spatial strategy.

When we analysed the subjects' first choices, we found that 8 subjects ( 3 bonobos, 3 chimpanzees, 2 orang utans) made feature choices, 11 subjects ( 2 bonobos, 3 chimpanzees, 2 gorillas, 4 orang utans) place choices, and 1 subject (1 gorilla) made a middle choice. There was no significant difference in the distribution of the first choices of the four species (chi-square test: $\chi^{2}=8.038, \mathrm{df}=6, P=0.243$ ).

Figure 6 depicts the mean percentage of choices in the test session of the experimental group and the control group. We found no significant difference between groups with respect to the percentage of feature and place choices (Mann-Whitney $U$ tests: feature: $N=11, U=9.500$, $P=0.340$; place: $N=11, U=9.500, P=0.340$ ). This suggests that there was no effect of the number of distinct containers on feature and places choices in the two chimpanzee groups.

When we compared the percentage of feature and place choices in Experiment 1 and Experiment 2, we found that subjects made significantly more place choices in

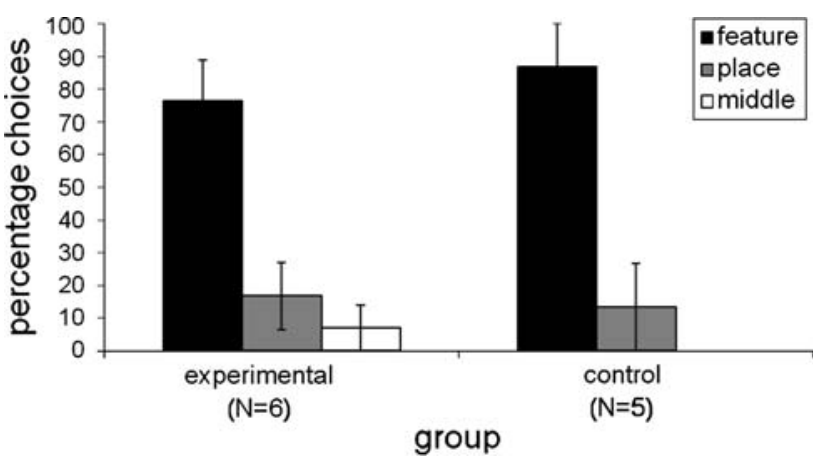

Fig. 6 Mean percentage of feature, place and middle choices $( \pm S E)$ of the experimental and the control group in Experiment 2. The experimental group was tested with three containers (two identical ones and one distinct one), and the control group was tested with three distinct containers

Experiment 2 than in Experiment 1 (Wilcoxon tests: feature: $Z=1.424, P=0.164$; place: $Z=2.521, P=0.009$ ). Feature and place choices on the platform did not differ significantly between subjects that first participated in Experiment 1 (see Table 1 for details) and subjects that first participated in Experiment 2 (Mann-Whitney $U$ tests: feature: $N=20, U=25.500, P=0.062$; place: $N=20$, $U=26.000, P=0.070$ ). In addition, feature and place choices on the floor (i.e. in Experiment 1) did not differ significantly between subjects that first participated in Experiment 1 and those that first participated in Experiment 2 (Mann-Whitney $U$ tests: feature: $N=17, U=30.000$, $P=0.784$; place: $N=17, U=29.500, P=0.896$ ).

\section{Discussion}

In our experiment, apes on average preferred to use a feature strategy when they had to indicate the location of a hidden food reward on a platform in front of them. After an initial training period where apes learnt to associate a food reward with a location and a container on a platform, 12 apes used a feature and 5 apes used a spatial strategy when they were tested with conflicting cues. Again, we found no differences in performance between the four great ape species.

Contrary to what we had expected based on the findings by Haun et al. (2006), apes did not prefer to use a spatial strategy. However, unlike the clear cut preference for the feature strategy we found in the first experiment, in the current experiment we found that five subjects used a spatial strategy to solve the task. We could rule out that this preference for the spatial strategy was linked to sex or age as in some other species were it has been shown for example that males preferentially rely on spatial strategies or use spatial information more accurately (e.g. chicks: Vallortigara 1996; humans: Postma et al. 2004). When we compared the percentage of feature and place choices in Experiment 1 
and Experiment 2, we found evidence that the percentage of place choices increased significantly from Experiment 1 to Experiment 2. These results demonstrate that the different tasks in Experiment 1 and Experiment 2 had some influence on which strategies were preferentially used by the apes. The results also show that at least some subjects switched between strategies, which indicates that apes are able to use the two strategies in a flexible way. In summary, reducing the number of rewarded locations, as well as preventing the apes from moving around during their search, seemed to have an effect on which strategy some subjects preferentially used.

One might argue that our findings diverged from those of Haun et al. (2006) because in our set-up we used two identical and one distinct container instead of three distinct containers. Thus, in our case, the relevant feature stimulus only had to compete with one instead of two other irrelevant stimuli, which might have increased the salience of that (feature) stimulus. Our control group, however, allowed us to rule out this possibility with respect to the chimpanzees' performance (other species could not be tested, due to the small sample sizes). We did not find a difference in performance between a chimpanzee group that was tested with two identical and one distinct containers and a chimpanzee group that was tested with three distinct containers, and, so we can assume that the number of distinct containers did not play a role in influencing strategy preferences in chimpanzees.

However, one other methodological difference between our experiment and the Haun et al. (2006) study remains that may account for the different findings. In our experiment we trained the subjects to choose the correct container prior to conflicting the feature and the place cues, whereas in the study by Haun et al. (2006) the subjects directly observed the food item being hidden under one container. Thus, in our experiment, the subjects were exposed to the same stimuli repeatedly while in the Haun et al. (2006) study the subjects were only briefly exposed to the stimuli. Furthermore, in our experiment the subjects initially had to learn the correct response, whereas in the study by Haun et al. (2006) they received visual information about where the reward was hidden. In order to investigate whether the absence and/or presence of training can account for the divergent findings, we conducted another experiment.

\section{Experiment 3}

The goal of Experiment 3 was to investigate how training prior to testing apes with conflicting cues may influence strategy preferences in apes. Apes were split into three different groups: the first group was tested with prior training in a replication of Experiment 2. A second group only observed the hiding of the food but without training. A third group was trained beforehand and then observed the hiding during the test phase. If training had the hypothesized effect, we would expect the following: Apes in the first group would preferentially use a feature strategy as in Experiment 2; and apes in the second group would preferentially use a spatial strategy as in Haun et al. (2006). If training and observing the hiding of the food had contrary effects, then we would expect that apes in the third group would show no preference for any of the two strategies.

Methods

\section{Subjects}

Fifteen subjects ( 5 bonobos, 10 chimpanzees) from Experiment 2 and six additional subjects ( 6 chimpanzees) that were naïve to the task participated in the current experiment. We used a between-subjects design and assigned the subjects to three different experimental groups: eight subjects (6 chimpanzees, 2 bonobos) were assigned to the 'learning' group, five subjects ( 3 chimpanzees, 2 bonobos) to the 'learning \& seeing' group, and eight subjects (7 chimpanzees, 1 bonobo) to the 'seeing' group (see Table 1).

\section{Materials}

For each of the three experimental groups, we used the following set of containers that differed in shape, size, and colour: a black square-shaped container, a yellow cup, and a pink round, flat container (see Fig. 3b). For each group, we counterbalanced the side on the platform and the container that were rewarded, so that e.g. one subject would be trained to find the food under the yellow cup on its right-hand side and another subject to find the food under the black square-shaped container on its left-hand side.

\section{Procedure}

Subjects in the 'learning group' were trained with the same procedure as in Experiment 2. In the test session, the subjects experienced 6 training trials followed by 12 test trials. Similar to Experiment 2, during test trials all containers were baited out of sight of the subjects to avoid differential reinforcement. At the beginning of each of the 12 test trials, we presented the subjects with the three containers on the platform in the same positions as during training. We pushed the platform with the containers only halfway towards the subjects and held a piece of food above the containers for about $3 \mathrm{~s}$. Then, we lowered an occluder and switched the positions of the containers on the right and left-hand side. After raising the occluder, we allowed the 
subjects to choose one of the three containers. This was repeated for 12 trials.

Subjects in the 'learning and seeing' group were trained and tested with the same procedure as described for the 'learning' group with the following exception. In the test session, after having pushed the platform halfway towards the subjects, we placed a piece of food under the container that was rewarded during training. This was done in full view of the subjects and lasted about $3 \mathrm{~s}$.

Subjects in the 'seeing' group were tested with the same procedure as subjects in the 'learning and seeing' group, with the exception that the apes did not receive any training and directly participated in one test session of 12 trials.

For all groups, we used banana slices as rewards with the exception of one subject, Fraukje, who received half a pellet.

\section{Data scoring and analysis}

We used live coding and coding from videotapes to code the following dependent variables: choice of the container under which the food could previously be found (feature choice); choice of the container in the place where the food could be found during training (place choice); choice of the container in the middle of the platform (middle choice) (see Fig. 4b).

As the data did not fulfil some of the requirements for using parametric statistics, all analyses were conducted with non-parametric tests. We first determined whether there was a significant difference in performance between the naïve and the experienced subjects in the 'learning' and in the 'seeing' group using Mann-Whitney $U$ tests. In order to test whether the different treatments had an influence on the subjects' choice behaviour, we tested whether there was a difference in performance between the three experimental groups by means of Kruskal-Wallis $H$ tests. In addition, we used one-sample Wilcoxon tests to determine whether on average subjects in each of the three groups made feature or place choices significantly above chance. Furthermore, we analysed the first choices of the subjects from all three experimental groups using chi-square tests. Finally, focusing on individual data we assessed whether individual subjects searched the containers randomly or whether they preferred to use either a feature or a spatial strategy using binomial tests (chance level 0.33). All statistical tests were evaluated with a significance level of 0.05 and exact, twotailed $P$ values are reported where applicable.

\section{Results}

The subjects of the 'learning' group needed on average $2.1 \pm 0.4$ sessions (Mean $\pm \mathrm{SE}$ ) and the subjects of the 'learning and seeing' group $3 \pm 0.9$ sessions (Mean $\pm \mathrm{SE}$ )

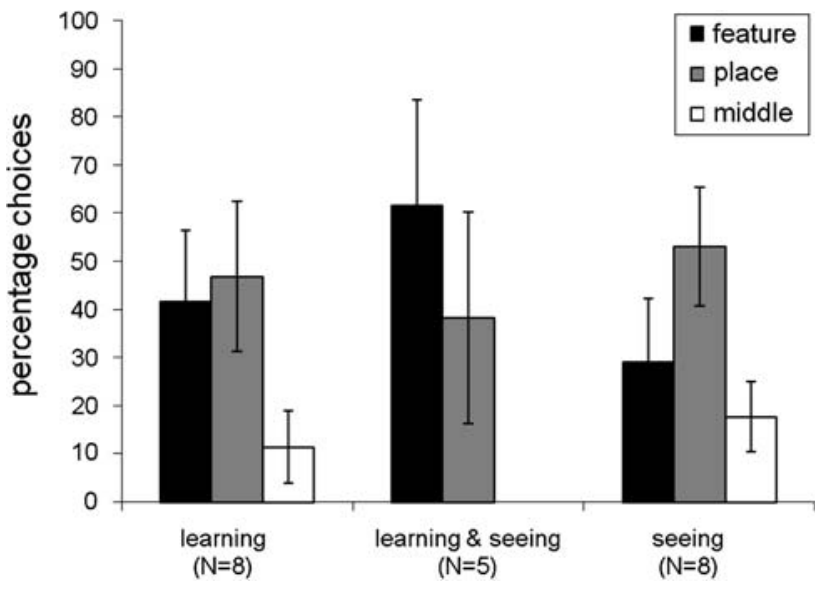

Fig. 7 Mean percentage of feature, place and middle choices $( \pm$ SE) of the three experimental groups in Experiment 3. The 'learning' group was trained to find the reward under one container in one location before they were tested with conflicting cues. The 'learning and seeing' group was first trained and then observed the reward being hidden during the test. Finally, the 'seeing' group never received training and only observed the reward being hidden during the test

to reach the training criterion. We found no significant difference in choice behaviour between naïve and experienced subjects in the 'learning' group (Mann-Whitney $U$ tests: feature: $N=8, U=2.000, P=0.107$; place: $N=8$, $U=4.000, P=0.357$ ) and the 'seeing' group (Mann-Whitney $U$ tests: feature: $N=8, U=6.500, P=0.821$; place: $N=8, U=3.000, P=0.214$ ). Therefore we combined the data from both naïve and experienced subjects for further analysis.

Figure 7 depicts the mean percentages of feature and place choices of the three experimental groups. We found no significant difference in the percentages of feature and place choices between the three groups (Kruskal-Wallis $H$ tests: feature: $\chi^{2}=1.255, p=0.546$; place: $\chi^{2}=0.453$, $P=0.807$ ). Focusing on whether the feature and place choices of each experimental group deviated from chance, we did not find a significant deviation for the 'learning' group (Wilcoxon tests: feature: $Z=0.281, P=0.820$; place: $Z=0.845, P=0.438$ ), for the the 'learning and seeing' group (feature: $Z=1.219, p=0.313$; place: $Z=0.406$, $P=0.750$ ), or for the 'seeing' group (feature: $Z=0.421$, $P=0.703$; place: $Z=0.1481, P=0.086$ ). Analysis of differences in first feature, place and middle choices of the three groups revealed no significant difference (chi-square: $\chi^{2}=3.602, \mathrm{df}=4, P=0.559$ ). That is, strategy preferences did not differ significantly, irrespective of whether subjects were trained or not or whether they observed the hiding of the reward or not.

Focusing on the individual data, we found that in the 'learning' group two of eight subjects used a feature strategy and three subjects used a spatial strategy (see Table 1). In the 'learning and seeing' group, three of the five subjects 
used a feature strategy and two subjects used a spatial strategy. Finally, in the 'seeing' group, two of eight subjects used a feature strategy and three subjects used a spatial strategy. In both the 'learning' and the 'seeing' groups, three subjects did not use any strategy at all.

\section{Discussion}

In this experiment, we found no significant difference in the choice behaviour of the three experimental groups when they were tested with conflicting cues on a platform in front of them. In addition, we did not find any significant preference for feature or spatial strategies in any of the three groups. Focusing on individual strategies, we found that subjects in all three experimental groups employed feature, as well as spatial strategies. Even some subjects of the 'seeing' group, who lacked training and had the least exposure to the stimuli, used a feature-based strategy. Among the three different groups, the 'seeing' group was tested with a design that was most comparable to the design of Haun et al. (2006), so that our results indicate that the feature cues might be more readily used than previously described.

Our findings also show, that irrespective of whether subjects were trained or not or observed the hiding of the reward or not, there was a high degree of inter-individual variation. In two cases, we also observed that subjects of the 'learning' group (Joey, Sandra), who were tested with the exact same procedure as in Experiment 2, changed their strategies from Experiment 2 to Experiment 3. Moreover, subjects in the 'learning' group did not show an overall preference for a feature strategy as we had found in Experiment 2 . This may indicate that apes' strategy preferences are unstable and subject to change over time.

The data from the current experiment does not allow us to draw any final conclusions as to why apes preferred to use feature strategies in Experiment 2 and spatial strategies in Haun et al. (2006). One might argue that the strategy preferences in Experiment 3 were influenced by the fact that the majority of subjects was already familiar with the task from participating in previous studies. We can partly rule out this possibility because we found no significant difference in choice behaviour between experienced (i.e. subjects that participated in Experiment 1 and/or Experiment 2) and naive subjects. However, one factor that may have played a role is the small sample size of subjects that was tested in the three experimental conditions. While in Experiment 2 and in the study by Haun et al. (2006), 20 and 25 subjects were tested, respectively; in Experiment 3, a maximum of 8 subjects was tested per experimental condition. Limitations in sample size may have prevented us from detecting any significant differences in strategy preferences in the three groups. In addition to these limitations, we cannot entirely rule out that other methodological differences in the design of our experiment and the one by Haun et al. (2006) might have played a role in shaping strategy preferences. For instance, the location of the reward changed from trial to trial in the study by Haun et al. (2006), whereas the reward location remained constant in our experiment. Further investigations could explore how the spatial stability of a resource (i.e. whether it can always be found in the same location or whether it can be found in different locations) influences strategy preferences.

\section{General discussion}

We found in three studies that apes can use different strategies to remember the location of hidden food items. In a task, where apes had to remember 12 food locations (that were contiguous with one feature cue) and could move around in the search space they preferred to use a feature strategy. In a task, where they had to remember one food location on a platform in front of them, they still preferred to use a feature strategy, but some individuals used a spatial strategy. Finally, when apes were tested with different variations of the task on the platform (i.e. received training beforehand or not; or observed the hiding of the reward or not), they used both strategies irrespective of experimental conditions and exhibited some individual variation in strategy preference.

While the study by Haun et al. (2006) had shown that apes preferentially use spatial strategies, we showed that in a similar spatial memory tasks they preferred to use feature strategies. This demonstrates that apes are capable of using both strategies but that strategy preference seems to be shaped by a number of different factors. For example, we found evidence that the number of search locations may play a role as some apes used a spatial strategy when they had to remember one food location (and one feature cue) but used a feature strategy when they had to remember 12 food locations (and one feature cue). Increasing the number of search locations while keeping the number of feature cues constant possibly increased the relative salience of the feature cues. Similarly, it was shown in spatial reorientation tasks that increases in the size of the search space and thus possibly increases in the salience of the features cues had an influence on which cues were preferentially used. In these tasks, subjects switched from solely using spatial cues (i.e. the geometry of the room) to using spatial and feature cues (e.g. children: Learmonth et al. 2002; fish: Sovrano et al. 2007).

In addition, we hypothesized that prior training and, thus, repeated exposure to the stimuli may influence strategy preference, but we could not provide conclusive evidence for this claim. Yet, Hodgson and Healy (2005) used a 
similar argument to explain cue preferences in a non-storing bird species (great tits). The authors found that great tits preferred to use spatial cues-contrary to what had been found in previous experiments (Clayton and Krebs 1994). However, more systematic experiments are needed to support the claim that experience may shape cue preference.

In our studies, we brought two different cues into conflict in order to determine which cue apes preferentially used. Given that under natural circumstances cues are abundant, it is a question for further investigation how cues from different sources of information are processed in order to remember places of interest such as food sources, nests, water holes, etc. For example, if animals are given a choice between a location that is indicated by two or three cues and a location that is indicated by only one cue, which location will they choose? Are animals able to integrate different sources of information or do they rely on one dominant cue? In the case of squirrels, it was demonstrated that they would choose a location that was indicated by the majority of cues over a location that was only indicated by one cue (Gibbs et al. 2007; Waisman and Jacobs 2008). Thus, squirrels seem to be able to integrate different sources of information. Future research in great apes' spatial memory could follow this path and investigate how great apes process multiple cues.

Acknowledgments We thank Natacha Mendes, Daniel Haun and Conny Schrauf and the anonymous reviewers for providing helpful comments on an earlier version of the manuscript and Anna-Claire Schneider for improving the English of the manuscript. The reported experiments comply with all laws of the country in which they were performed.

Open Access This article is distributed under the terms of the Creative Commons Attribution Noncommercial License which permits any noncommercial use, distribution, and reproduction in any medium, provided the original author(s) and source are credited.

\section{References}

Astié AA, Kacelnik A, Reboreda JC (1998) Sexual differences in memory in shiny cowbirds. Anim Cogn 1:77-82

Brodbeck DR (1994) Memory for spatial and local cues: a comparison of a storing and a non- storing species. Learn Behav 22:119-133

Brodbeck DR, Shettleworth SJ (1995) Matching location and color of a compound stimulus: comparison of a food-storing and a nonstoring bird species. J Exp Psych Anim Behav 21:64-77

Cheng K (1986) A purely geometric module in the rat's spatial representation. Cognition 23:149-178

Cheng K, Newcombe N (2005) Is there a geometric module for spatial orientation? Squaring theory and evidence. Psychon Rev 12:1-23

Clayton NS, Krebs JR (1994) Memory for spatial and object-specific cues in food-storing and non-storing birds. J Comp Physiol A 174:371-379

Day LB, Ismail N, Wilczynski W (2003) Use of position and feature cues in discrimination learning by whiptail lizard (Cnemidophorus inornatus). J Comp Psychol 117:440-448

Dumas C (1998) Figurative and spatial information and search behavior in dogs (Canis familiaris). Behav Process 42:101-106
Gibbs SEB, Lea SEG, Jacobs LF (2007) Flexible use of spatial cues in southern flying squirrel. Anim Cogn 10:203-209

Gouteux S, Thinus-Blanc C, Vauclair J (2001) Rhesus monkeys use geometric and nongeometric information during a reorientation task. J Exp Psychol Gen 130:505-519

Haun DBM, Call J, Janzen G, Levinson SC (2006) Evolutionary psychology of spatial representation in the hominidae. Curr Biol 16:1736-1740

Hermer L, Spelke ES (1994) A geometric process for spatial reorientation in young children. Nature 370:57-59

Hodgson ZG, Healy SD (2005) Preference for spatial cues in a nonstoring songbird species. Anim Cogn 8:211-214

Kelly DM, Spetch ML, Heth CD (1998) Pigeons' (Columba livia) Encoding of Geometric and Featural Properties of a Spatial Environment. J Comp Psychol 112:259-269

Learmonth AE, Nadel L, Newcombe NS (2002) Children's use of landmarks: implications for modularity theory. Psychol Sci 13:337-341

Lee SA, Shusterman A, Spelke ES (2006) Reorientation and landmarkguided search by young children. Psychol Sci 17:577-582

Newcombe NS, Ratliff KR (2007) Explaining the development of spatial reorientation: modularity-plus-language versus the emergence of adaptive combination. In: Plumert J, Spencer J (eds) The emerging spatial mind. Oxford University Press, New York

Postma A, Winkel J, Tuiten A, van Honk J (1999) Sex differences and menstrual cycle effects in human spatial memory. Psychoneuroendocrino 24:175-192

Postma A, Jager G, Kessels RPC, Koppeschaar HPF, van Honk J (2004) Sex differences for selective forms of spatial memory. Brain Cognition 54:24-34

Regolin L, Garzotto B, Rugani R, Pagni P, Vallortigara G (2005) Working memory in the chick: parallel and lateralized mechanisms for encoding of object- and position- specific information. Behav Brain Res 157:1-9

Shettleworth SJ (1998) Cognition, evolution and behaviour. Oxford University Press, USA

Sovrano VA, Bisazza A, Vallortigara G (2003) Modularity as a fish (Xenotoca eiseni) views it: conjoining geometric and nongeometric information for spatial reorientation. J Exp Psych Anim Behav 29:199-210

Sovrano VA, Bisazza A, Vallortigara G (2007) How fish do geometry in large and small spaces. Anim Cogn 10:47-54

Strasser R, Bingman VP (1996) The relative importance of location and feature cues for homing pigeons (Columba livia) goal recognition. J Comp Psychol 110:77-87

Tinklepaugh OL (1932) Multiple delayed reaction with chimpanzees and monkeys. J Comp Psychol 13:207-243

Vallortigara G (1996) Learning of colour and position cues in domestic chicks: males are better at position, females at colour. Behav Process 36:289-296

Vallortigara G (2000) Comparative neuropsychology of the dual brain: a stroll through animals' left and right perceptual worlds. Brain Lang 73:189-219

Vallortigara G, Zanforlin M, Pasti G (1990) Geometric modules in animals' spatial representations: a test with chicks (Gallus gallus domesticus). J Comp Psychol 104:248-254

Vallortigara G, Feruglio M, Sovrano VA (2005) Reorientation by geometric and landmark information in environments of different size. Developmental Sci 8:393-401

Vargas JP, Lopéz JC, Salas C, Thinus-Blanc C (2004) Encoding of geometric and featural spatial information by goldfish (Carassius auratus). J Comp Psychol 118:206-216

Waisman AS, Jacobs LF (2008) Flexibility of cue use in the fox squirrel. Anim Cogn 11:625-636

Williams JT (1967) A test for dominance of cues during maze learning by toads. Psychon Sci 9:259-260 